
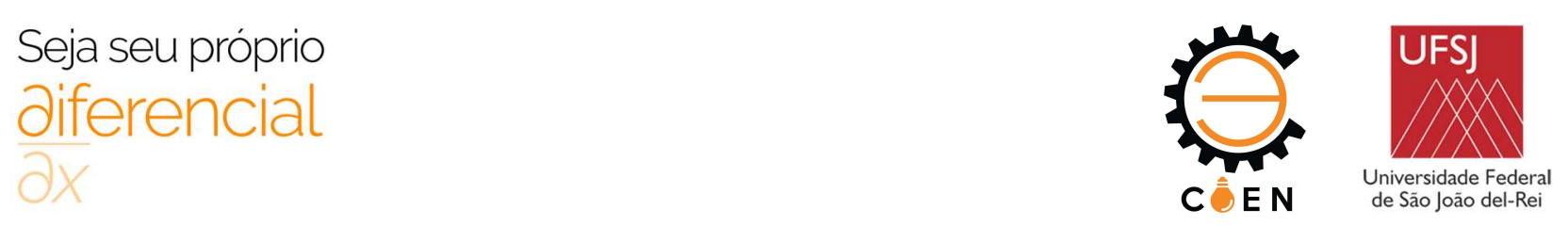

\title{
APLICAÇÃO DE TÉCNICAS DE MANUTENÇÃO PREDITIVA EM UMA BANCADA COM MOTOR ELÉTRICO MONOFÁSICO
}

\author{
Marcel Henrique Lopes ${ }^{(1)}$ (marcel.henrike@hotmail.com), Leonardo Vasconcelos Bessa ${ }^{(2)}$ \\ (leobessa96@gmail.com), Mariana Fernandes Souto ${ }^{(2)}$ (mariana.fsouto@hotmail.com), \\ Jorge Nei Brito ${ }^{(1)}$ (brito@ufsj.edu.br) \\ (1) Universidade Federal de São João Dei Rei (UFSJ); Departamento de Engenharia Mecânica \\ (2) Universidade Federal de São João Dei Rei (UFSJ); Departamento de Engenharia Elétrica
}

RESUMO: Manutenção preditiva é o acompanhamento de máquinas equipamentos através de dados coletados por meio de monitoramento ou inspeções. Através de técnicas de manutenção foram utilizados ensaios não destrutivos como a análise sem contato, a termografia infravermelha forma imagens térmicas chamadas de termogramas, e através da Análise de Forma de Deflexão Operacional (Operating Deflection Shape - ODS) é possivel obter o comportamento dinâmico e vibracional de operação de uma bancada didática. Os resultados obtidos possibilitaram realizar o mapeamento térmico do motor em funcionamento, as falhas mecânicas e estruturais e os efeitos da transmissibilidade de vibração. Este estudo pode ser aplicado em ambientes industriais estabelecendo uma correção mais rápida e assertiva, indicando ações corretivas que podem eliminar falhas no estágio inicial.

PALAVRAS-CHAVE: Manutenção Preditiva, Análise termográfica, Análise de Vibração, Forma de Deflexão Operacional.

\section{INTRODUÇÃO}

Manutenção preditiva é definida como manutenção que permite garantir uma qualidade de serviço desejada, com base na aplicação de técnicas de análise, utilizando-se meios de supervisão ou de amostragem (OLIVEIRA, 2012).

Há algum tempo a utilização de ensaios não destrutivos têm se mostrado como importante ferramenta para o controle do desempenho dos materiais em serviço. Esses ensaios são definidos como sendo aqueles que não prejudicam nem causam danos ao uso futuro do elemento ensaiado, ou seja, não provocam perdas na capacidade resistente do elemento (PEDRA, 2011).

Dentre as técnicas de análise não destrutiva, a Análise de Vibração e Termografia Infravermelha têm sido utilizadas para aplicações em diversos campos de atuação. A termografia é a técnica preditiva que permite o acompanhamento dada temperatura e a formação de imagens térmicas, denominada de termogramas. A temperatura é um dos parâmetros de mais fácil compreensão, e o acompanhamento da sua variação é muito importante, tanto para equipamentos elétricos como mecânicos, pois permite constatar uma alteração na condição dosdos equipamentos, componentes e do próprio processo como um todo (SOUZA, 2011).

Segundo Souza (2014), a Análise Modal Experimental (AME) é um conjunto de técnicas experimentais e modelos utilizados para identificação de parâmetros modais em estruturas, máquinas e equipamentos. Ela segue uma ordem inversa a análise teórica. E recebe este nome quando os parâmetros modais são identificados conhecendo-se a força de excitação e as técnicas empregadas.

A análise da Forma de Deflexão Operacional, do inglês Operating Deflection Shape (ODS), analisa a forma como uma estrutura vibra em um determinado instante de tempo ou frequência. 
Um modo operacional pode ser qualquer movimento forçado de dois ou mais corpos da estrutura que ao serem especificados definem uma forma. A frequência de vibração não coincide necessariamente com uma das frequências naturais do sistema. Em uma frequência qualquer, o modo operacional será uma combinação linear dos modos próprios do sistema, contendo a contribuição de todos os modos (GEVINSKI, 2014).

A ODS é a maneira mais simples de ver como uma máquina ou estrutura se move durante sua operação, seja em uma frequência ou momento específico no tempo (Vibrant Technology, Inc., 2016). O estudo por ODS é um teste de vibração e procedimento de análise capaz de fornecer a forma de deformação em resposta às forças aplicadas à estrutura e, também, de produzir animações das características de deformação de um sistema mecânico vibratório.

\section{MATERIAIS E MÉTODOS}

A bancada experimental utilizada, Figura 1, é composta de um motor de indução monofásico de $2 \mathrm{CV}, 3480 \mathrm{rpm}, 110 / 220 \mathrm{~V}$. Os testes experimentas foram realizados no Laboratório de Sistemas Dinâmicos (LASID), do Departamento de Engenharia Mecânica (DEMEC), da Universidade Federal de São João del-Rei(UFSJ).

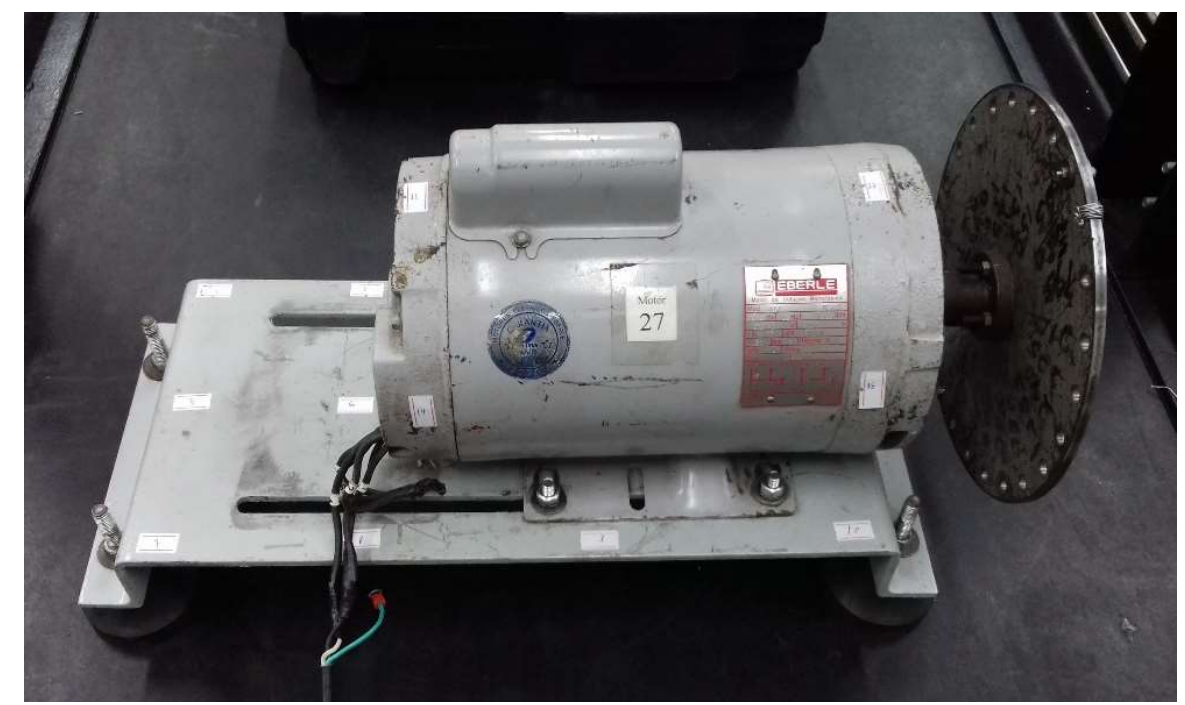

Figura 1. Bancada de testes Fonte: Autor (2019).

A metodologia para a análise do sistema dinâmico consiste na medição da Forma de Deflexão Operacional (ODS) com o motor em funcionamento. Para a coleta da vibração utilizou-se o coletor/analisador de dados SKF Microlog-GX $75^{\circledR}$, Figura 2.

Os sinais foram coletados com o acelerômetro SKF CMSS 2200, sensibilidade de $100 \mathrm{mV}$, colocado alternadamente nas posições: vertical, horizontal e axial; em pontos previamente definidos. Posteriormente os dados coletados foram analisados no software ME'scopeVESTM, tecnologia da Vibrant Techonology, o qual possui uma família de pacotes que auxiliam na observação, análise, e documentação de problemas relacionados a ruídos e vibração em máquinas e estruturas. 


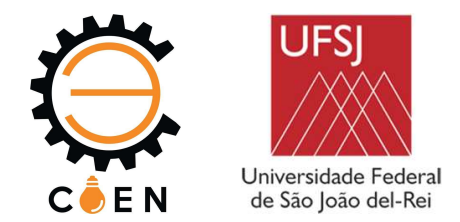

O ME'scopeVESTM, pode ser usado para processamento de sinais através da análise ODS, AME, AMO, Análise Acústica e Vibro Acústica, dentre outros. Para a Análise Termográfica utilizouse a câmera termográfica Modelo Flir T200, Figura 3.

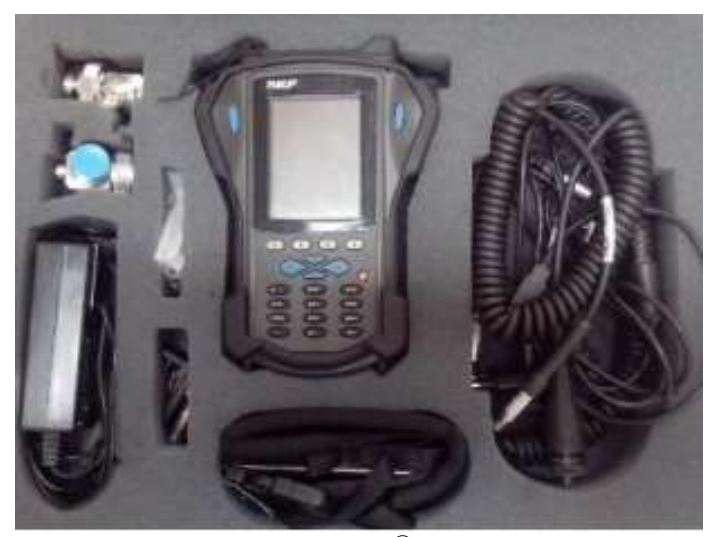

Figura 2. Microlog-GX $75^{\circledR}$. Fonte: Autor (2019).

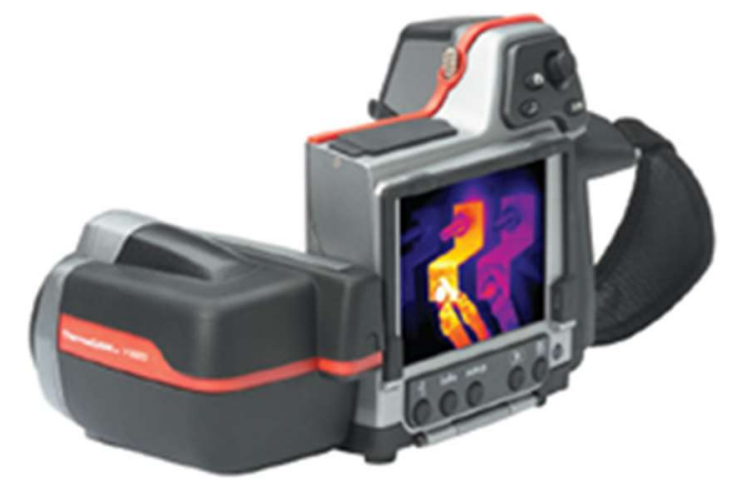

Figura 3. Câmera termográfica Flir T200. Fonte: Autor (2019).

A análise do sistema em estudo, é realizada pela medição da malha que é discretizada igualmente no sistema real e na estrutura desenvolvida no software para a análise, Figura 4. Optouse por coletar 15 pontos na bancada de teste, devido as dimensões da estrutura em questão, com a sua direção de liberdade, X,Y ou Z.



Figura 4. Pontos de coleta da bancada. Fonte: Autor (2019).

\section{ANÁLISE DOS RESULTADOS}

Através do termograma, Figura 5, foi possível caracterizar e mapear os pontos de maior temperatura do motor em funcionamento. A análise teve um foco entre as frequências de $0 \mathrm{a} 100 \mathrm{~Hz}$. Nessa faixa a frequência de excitação promovida pelo primeiro harmônico é de $58,8 \mathrm{~Hz}$. 


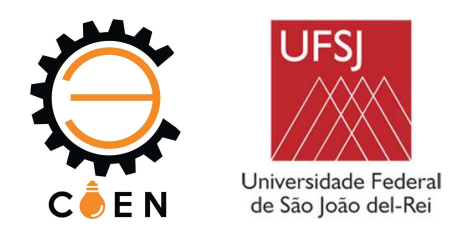

Na Figura 5 é possível observar o espectro de todos os pontos sobrepostos, apresentando baixas variações decorrentes da transmissibilidade das forças vibratórias, e formando um padrão em seu primeiro harmônico. Tal característica é esperada devido a frequência de rotação do motor, se localizar, nesse ponto em específico.

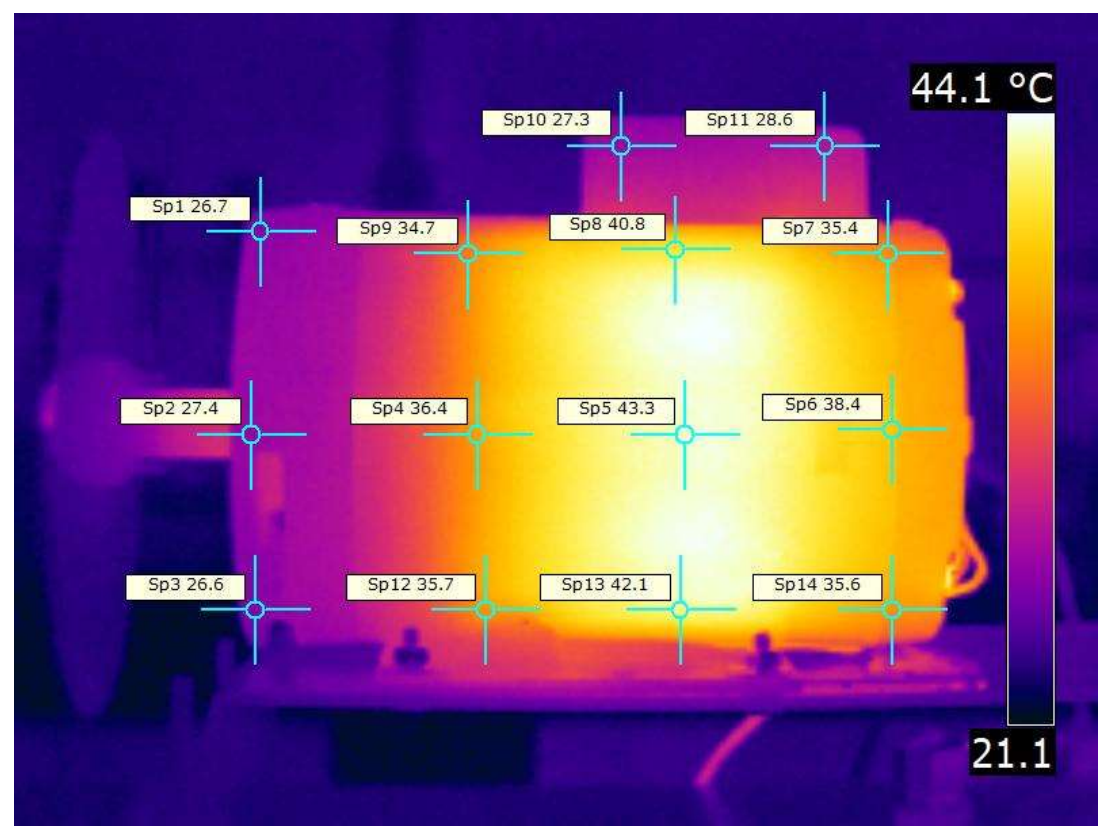

Figura 5. Termograma do motor de indução monofásico. Fonte: Autor (2019).

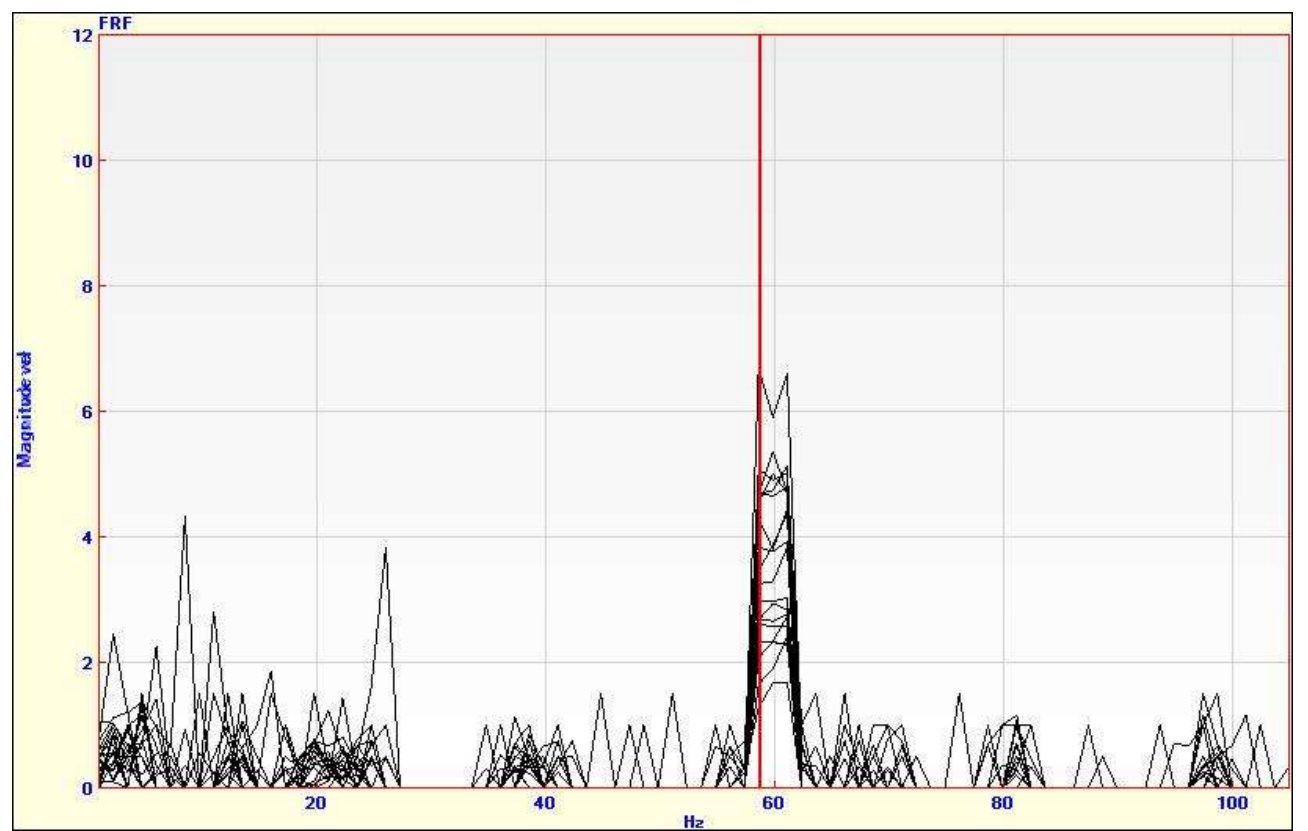

Figura 6. Espectro FRF de todos os pontos coletados sobrepostos. Fonte: Autor (2019).

IX COEN - Congresso de Engenharias da UFSJ Interconexão. 


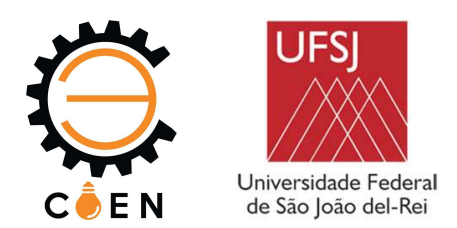

Na Figura 7 são apresentados o modelo computacional e o comportamento decorrente das excitações sofridas na estrutura.

Nota-se que durante a movimentação da estrutura houve uma maior movimentação na lateral direita do motor, sendo como possível consequência o sentido da rotação do motor, e do disco de balanceamento com as massas inseridas, a fim de deixá-lo balanceado.

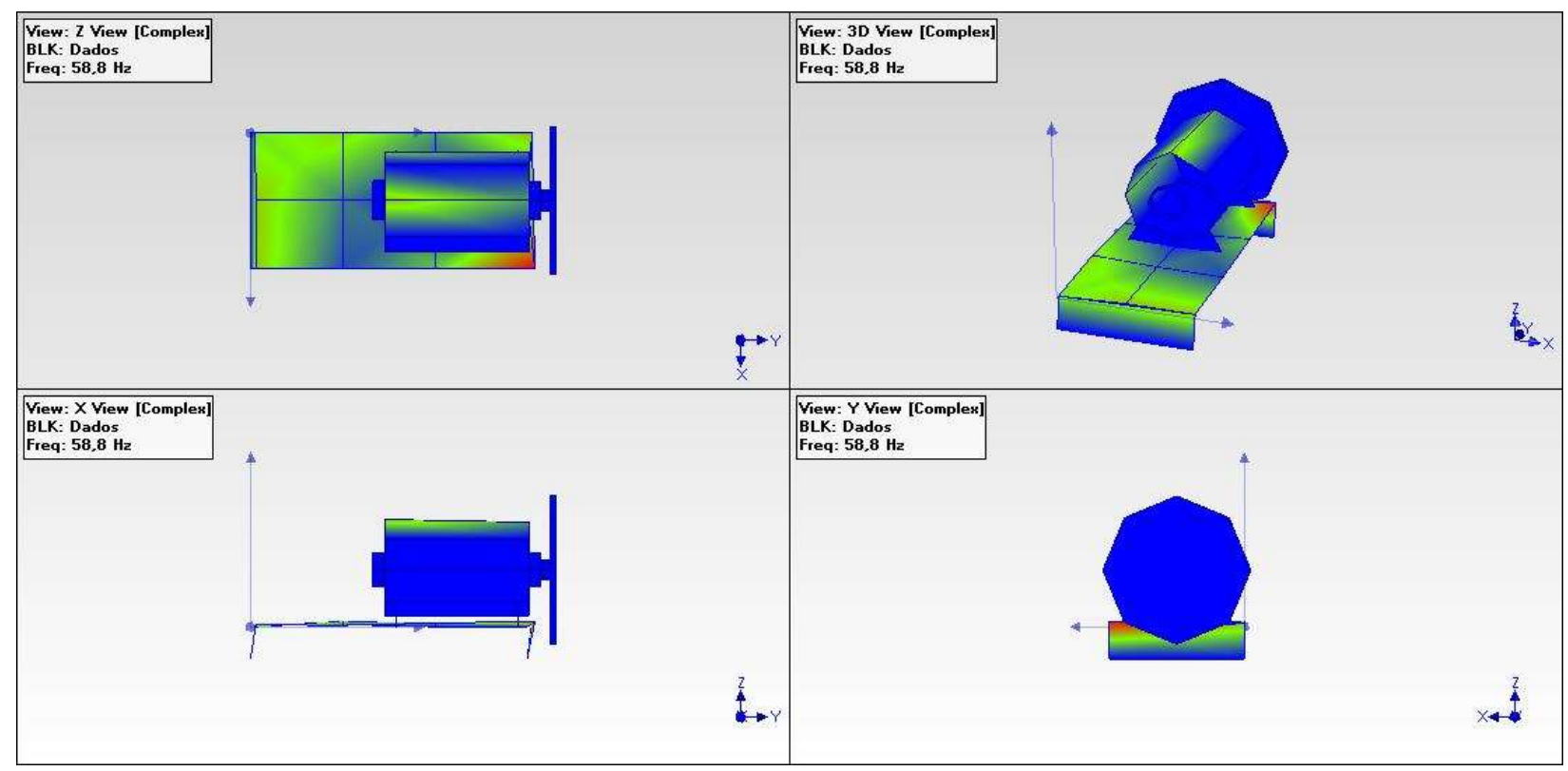

Figura 7. Simulação das excitações na estrutura. Fonte: Autor (2019).

\section{CONCLUSÃO}

Com base nos resultados encontrados, o método utilizado para detecção de falha mecânicas se prova eficaz e sendo de fundamental importância em aplicações industriais, cuja as análises de falhas são essenciais para diagnóstico e tratamento de forma a garantir a durabilidade do equipamento. As análises realizadas mapeiam as falhas mecânicas e pontos frágeis da estrutura permitindo realizar um reforço, garantido maior assertividade e redução no tempo médio de reparo da máquina. Durante o estudo do comportamento vibracional da estrutura é possível avaliar como o sistema real se difere da teoria de vibrações.

Os resultados encontrados estão de acordo com o esperado, a fim de dar continuação nesse estudo, pretende-se realizar uma comparação utilizando a mesma técnica de manutenção, mas com a inserção de falhas mecânicas e elétricas.

\section{DIREITOS AUTORAIS}

Os autores são os únicos responsáveis pelo conteúdo das informações contidas neste artigo

\section{REFERÊNCIAS}

IX COEN - Congresso de Engenharias da UFSJ 
BASSI, Anderson Flávio. Análise da Perda energética no cilindro em motor diesel através da termografia. Dissertação de Pós-Graduação em Engenharia da Energia. Universidade Federal de São João Del Rei, 2012.

GEVINSKI, JAKERSON RICARDO. Determinação da deformação dinâmica em superfícies utilizando parâmetros vibracionais. Campinas - SP, 2014.

OLIVEIRA, Davi Borges de. Manutenção preditiva; Termografia; Indústria sucroalcooleira; Manutenção preditiva; Termografia; Indústria sucroalcooleira. Trabalho de Conclusão de Curso apresentado ao Centro Universitário de Formiga - UNIFOR-MG, 2012.

PEDRA, SOLANGE ANDERE. Potencialidades da termografia infravermelha aplicada ao design do conforto térmico de alvenaria estrutural. Dissertação de Mestrado, Universidade do Estado de Minas Gerais, UEMG. 2011.

SOUZA, P. H. M. Análise Modal Experimental Utilizando Vibrômetro Laser Doppler. Trabalho de Conclusão de Curso - Departamento do Curso de Engenharia Mecânica - Universidade Federal de São João Del Rei, 57 p., 2014.

SOUZA, VALDIR CARDOSO DE. Organização e gerenciamento da manutenção: programação e controle de manutenção / Valdir Cardoso de Souza. - 4a . Ed. - São Pauso: All Printe Editora. 2011.

VIBRANT TECHNOLOGY INC., Me`scopeVes, Série Visual Engenharia. Estados Unidos da América.

\title{
APPLICATION OF PREDICTIVE MAINTENANCE TECHNIQUES ON A WORKBENCH WITH A SINGLE-PHASE ELECTRIC MOTOR
}

\author{
Marcel Henrique Lopes ${ }^{(1)}$ (marcel.henrike@hotmail.com), Leonardo Vasconcelos Bessa ${ }^{(2)}$ \\ (leobessa96@gmail.com), Mariana Fernandes Souto ${ }^{(2)}$ (mariana.fsouto@hotmail.com), \\ Jorge Nei Brito ${ }^{(1)}$ (brito@ufsj.edu.br)
}

(1) Universidade Federal de São João Dei Rei (UFSJ); Mechanical Engineering Department

(2) Universidade Federal de São João Dei Rei (UFSJ); Department of Electrical Engineering

ABSTRACT: Predictive maintenance is the monitoring of machine equipment through data collected through monitoring or inspections. Through non-destructive tests such as non-contact analysis, infrared thermography forms thermal images called thermograms, and through Operating Deflection Shape (ODS) is possible to obtain the dynamic and vibrational behavior of a didactic workbench. The results obtained allowed to realize the thermal mapping of the motor in operation, the mechanical and structural failures and the effects of the transmissibility of vibration. This study can be applied in industrial environments, establishing a more rapid and assertive correction, indicating corrective actions that can eliminate failures in the initial stage.

KEYWORDS: Predictive Maintenance, Thermographic Analysis, Vibration Analysis, Operational Deflection Form. 